\title{
On lifts of projectable-projectable classical linear connections to the cotangent bundle
}

\begin{abstract}
We describe all $\mathcal{F}^{2} \mathcal{M}_{m_{1}, m_{2}, n_{1}, n_{2}}$-natural operators $D: Q_{\text {proj-proj }}^{\tau}$ $\rightsquigarrow Q T^{*}$ transforming projectable-projectable classical torsion-free linear connections $\nabla$ on fibred-fibred manifolds $Y$ into classical linear connections $D(\nabla)$ on cotangent bundles $T^{*} Y$ of $Y$. We show that this problem can be reduced to finding $\mathcal{F}^{2} \mathcal{M}_{m_{1}, m_{2}, n_{1}, n_{2}}$-natural operators $D: Q_{\text {proj-proj }}^{\tau} \rightsquigarrow\left(T^{*}, \otimes^{p} T^{*} \otimes \otimes^{q} T\right)$ for $p=2, q=1$ and $p=3, q=0$.
\end{abstract}

1. Basic definitions and examples. A fibred-fibred manifold $Y$ is any commutative diagram

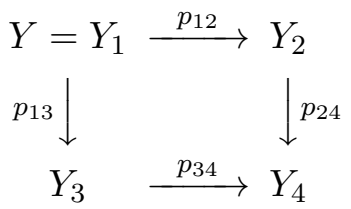

where maps $p_{12}, p_{13}, p_{24}, p_{34}$ are surjective submersions and an induced map $Y_{1} \rightarrow Y_{2} \times_{Y_{4}} Y_{3}, y \mapsto\left(p_{12}(y), p_{13}(y)\right)$ is a surjective submersion. A fibredfibred manifold has dimension $\left(m_{1}, m_{2}, n_{1}, n_{2}\right)$ if $\operatorname{dim} Y_{1}=m_{1}+m_{2}+n_{1}+n_{2}$, $\operatorname{dim} Y_{2}=m_{1}+m_{2}, \operatorname{dim} Y_{3}=m_{1}+n_{1}, \operatorname{dim} Y_{4}=m_{1}$. For two fibredfibred manifolds $Y, \widetilde{Y}$ of the same dimension $\left(m_{1}, m_{2}, n_{1}, n_{2}\right)$, a morphism $f: Y \rightarrow \widetilde{Y}$ is a quadruple of local diffeomorphisms $f_{1}: Y_{1} \rightarrow \widetilde{Y}_{1}, f_{2}: Y_{2} \rightarrow$

2010 Mathematics Subject Classification. 58A20, 58A32.

Key words and phrases. Fibred-fibred manifold, projectable-projectable linear connection, natural operator. 
$\tilde{Y}_{2}, f_{3}: Y_{3} \rightarrow \widetilde{Y}_{3}, f_{4}: Y_{4} \rightarrow \widetilde{Y}_{4}$ such that all squares of the cube in question are commutative, [2], [7].

All fibred-fibred manifolds of the given dimension $\left(m_{1}, m_{2}, n_{1}, n_{2}\right)$ and all their morphisms form the category which we denote by $\mathcal{F}^{2} \mathcal{M}_{m_{1}, m_{2}, n_{1}, n_{2}}$.

Every object from the category $\mathcal{F}^{2} \mathcal{M}_{m_{1}, m_{2}, n_{1}, n_{2}}$ is locally isomorphic to the standard fibred-fibred manifold

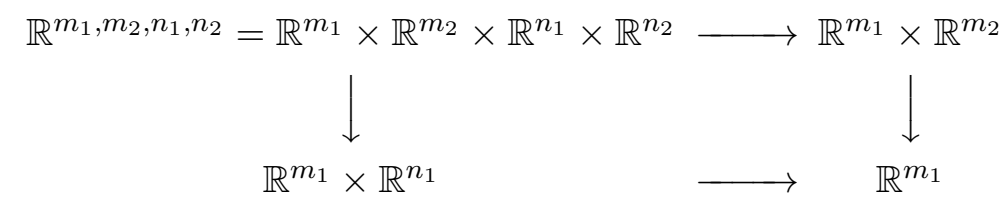

where arrows are obvious projections.

A classical linear connection $\nabla$ on a fibred-fibred manifold $Y$ is a tangent bundle homothety invariant section $\nabla: T Y \rightarrow J^{1} T Y$ of the 1-jet prolongation $J^{1} T Y \rightarrow T Y$ of the tangent bundle $T Y$. Recall that a classical linear connection $\nabla$ on $Y$ is called a projectable-projectable linear connection on a fibred-fibred manifold $Y$ if there exist classical linear connections $\nabla_{2}, \nabla_{3}$, $\nabla_{4}$ on $Y_{2}, Y_{3}, Y_{4}$, respectively, such that the connection $\nabla$ projects into $\nabla_{2}$ and $\nabla_{3}$ by maps $p_{12}$ and $p_{13}$, respectively, and connections $\nabla_{2}$ and $\nabla_{3}$ project into $\nabla_{4}$ by maps $p_{24}$ and $p_{34}$, respectively, [4], [1].

A classical linear connection $\nabla: T Y \rightarrow J^{1} T Y$ on $Y$ determines the corresponding covariant derivative $\nabla: \mathfrak{X}(Y) \times \mathfrak{X}(Y) \rightarrow \mathfrak{X}(Y)$ of vector fields on $Y$ satisfying the additional projectability-projectability condition.

We say that a classical linear connection $\nabla$ on a fibred-fibred manifold $Y$ is torsion-free if the torsion tensor $T(X, Y)$ of $\nabla$ vanishes, i.e. $T(X, Y)=$ $\nabla_{X} Y-\nabla_{Y} X-[X, Y]=0$.

In the present paper we consider a problem of constructing of a classical linear connection $D(\nabla)$ on the cotangent bundle $T^{*} Y$ of $Y$ by means of a projectable-projectable classical torsion-free linear connection $\nabla$ on an $\left(m_{1}, m_{2}, n_{1}, n_{2}\right)$-dimensional fibred-fibred manifold $Y$. To this aim we will consider a characterization of $\mathcal{F}^{2} \mathcal{M}_{m_{1}, m_{2}, n_{1}, n_{2}}$-natural operators $D$ : $Q_{\text {proj-proj }}^{\tau} \rightsquigarrow Q T^{*}$ corresponding to above constructions.

A similar problem in the case of usual $n$-dimensional manifolds $M$ and classical linear connections $\nabla$ (not necessarily torsion-free) was studied by M. Kureš [6] and it was extended to $\otimes^{k} T^{*} M$ in [5].

We will formulate definitions of natural operators which can be treated as special cases of the general concept of natural operators from [3].

Definition 1. An $\mathcal{F}^{2} \mathcal{M}_{m_{1}, m_{2}, n_{1}, n_{2}}$-natural operator $D: Q_{\text {proj-proj }}^{\tau} \rightsquigarrow Q T^{*}$ transforming projectable-projectable classical torsion-free linear connections $\nabla$ on $\left(m_{1}, m_{2}, n_{1}, n_{2}\right)$-dimensional fibred-fibred manifolds $Y$ into classical linear connections $D(\nabla)$ on $T^{*} Y$ is a family $D=\left(D_{Y}\right)$ of $\mathcal{F}^{2} \mathcal{M}_{m_{1}, m_{2}, n_{1}, n_{2}}{ }^{-}$ invariant regular operators

$$
D_{Y}: Q_{\text {proj-proj }}^{\tau}(Y) \rightarrow Q\left(T^{*} Y\right)
$$


for any fibred-fibred manifold $Y$ of the dimension $\left(m_{1}, m_{2}, n_{1}, n_{2}\right)$, where $Q_{\text {proj-proj }}^{\tau}(Y)$ is the set of all projectable-projectable classical torsion-free linear connections on the fibred-fibred manifold $Y$ and $Q\left(T^{*} Y\right)$ is the set of all classical linear connections (not necessarily torsion-free) on $T^{*} Y$. The $\mathcal{F}^{2} \mathcal{M}_{m_{1}, m_{2}, n_{1}, n_{2}}$-invariance of (the operator) $D$ means that if any projectable-projectable classical torsion-free linear connections $\nabla \in Q_{\text {proj-proj }}^{\tau}(Y)$, $\widetilde{\nabla} \in Q_{\text {proj-proj }}^{\tau}(\widetilde{Y})$ are $\varphi$-related by an $\mathcal{F}^{2} \mathcal{M}_{m_{1}, m_{2}, n_{1}, n_{2}}$-invariant map $\varphi: Y \rightarrow \widetilde{Y}$ (i.e. $J^{1} T \varphi \circ \nabla=\widetilde{\nabla} \circ T \varphi$ ) then induced classical linear connections $D(\nabla) \in Q\left(T^{*} Y\right)$ and $D(\widetilde{\nabla}) \in Q\left(T^{*} \widetilde{Y}\right)$ are $T^{*} \varphi$-related by $T^{*} \varphi: T^{*} Y \rightarrow$ $T^{*} \widetilde{Y}$ (i.e. $J^{1} T\left(T^{*} \varphi\right) \circ D(\nabla)=D(\widetilde{\nabla}) \circ T\left(T^{*} \varphi\right)$ ), where $T^{*} \varphi$ is a cotangent map to $\varphi$.

The regularity of $D$ means that $D$ transforms smoothly parameterized families of projectable-projectable classical torsion-free linear connections into smoothly parameterized families of classical linear connections.

Example 1. An example of $\mathcal{F}^{2} \mathcal{M}_{m_{1}, m_{2}, n_{1}, n_{2}}$-natural operator

$$
D: Q_{\text {proj-proj }}^{\tau} \rightsquigarrow Q T^{*}
$$

is a family $D^{T^{*}}=\left(D_{Y}^{T^{*}}\right)$ of operators

$$
D_{Y}^{T^{*}}: Q_{\text {proj-proj }}^{\tau}(Y) \rightarrow Q\left(T^{*} Y\right)
$$

given by the formula $D_{Y}^{T^{*}}(\nabla)=\nabla^{T^{*} Y}$, where $Y \in \operatorname{Obj}\left(\mathcal{F}^{2} \mathcal{M}_{m_{1}, m_{2}, n_{1}, n_{2}}\right)$, $\nabla \in Q_{\text {proj-proj }}^{\tau}(Y)$ and $\nabla^{T^{*} Y}$ is a horizontal lift of $\nabla$ on $Y$ to the cotangent bundle $T^{*} Y$.

We define a horizontal lift $\nabla^{T^{*} Y}$ of a projectable-projectable classical torsion-free linear connection $\nabla$ to the cotangent bundle $T^{*} Y$ as

$$
\nabla^{T^{*} Y}=\nabla^{C}-R_{Y}^{V}(\nabla)
$$

where $\nabla^{C}$ is the complete lift of $\nabla$ and $R_{Y}^{V}(\nabla)$ means the vertical lift of the curvature tensor $R_{Y}(\nabla)$ of $\nabla$, [8].

Definition 2. An $\mathcal{F}^{2} \mathcal{M}_{m_{1}, m_{2}, n_{1}, n_{2}}$-natural operator

$$
D: Q_{\text {proj-proj }}^{\tau} \rightsquigarrow\left(T^{*}, \otimes^{p} T^{*} \otimes \otimes^{q} T\right)
$$

transforming projectable-projectable classical torsion-free linear connections $\nabla$ on $\left(m_{1}, m_{2}, n_{1}, n_{2}\right)$-dimensional fibred-fibred manifolds $Y$ into fibred maps $D(\nabla): T^{*} Y \rightarrow \otimes^{p} T^{*} Y \otimes \otimes^{q} T Y$ covering the identity $i d_{Y}$ is a family of $\mathcal{F}^{2} \mathcal{M}_{m_{1}, m_{2}, n_{1}, n_{2}}$-invariant regular operators

$$
D=\left(D_{Y}\right): Q_{\text {proj-proj }}^{\tau}(Y) \rightarrow C_{Y}^{\infty}\left(T^{*} Y, \otimes^{p} T^{*} Y \otimes \otimes^{q} T Y\right)
$$

defined for any $\left(m_{1}, m_{2}, n_{1}, n_{2}\right)$-dimensional fibred-fibred manifold $Y$.

The $\mathcal{F}^{2} \mathcal{M}_{m_{1}, m_{2}, n_{1}, n_{2}}$-invariance of $D$ means that if two projectableprojectable classical torsion-free linear connections $\nabla \in Q_{\text {proj-proj }}^{\tau}(Y)$ and $\widetilde{\nabla} \in Q_{\text {proj-proj }}^{\tau}(\tilde{Y})$ are $\varphi$-related by an $\mathcal{F}^{2} \mathcal{M}_{m_{1}, m_{2}, n_{1}, n_{2}}$-map $\varphi: Y \rightarrow \widetilde{Y}$ 
then induced maps $D(\nabla): T^{*} Y \rightarrow \otimes^{p} T^{*} Y \otimes \otimes^{q} T Y$ and $D(\widetilde{\nabla}): T^{*} \widetilde{Y} \rightarrow$ $\otimes^{p} T^{*} \widetilde{Y} \otimes \otimes^{q} T \widetilde{Y}$ are $\varphi$-related, i.e. the following diagram is commutative

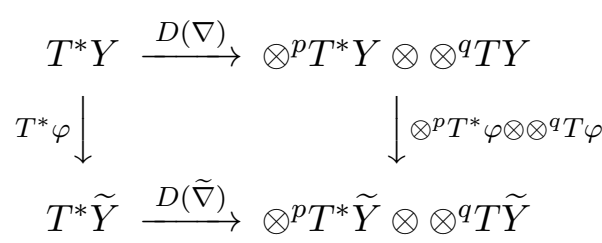

where $T \varphi: T Y \rightarrow T \tilde{Y}$ is a tangent map to $\varphi: Y \rightarrow \widetilde{Y}$ and $T^{*} \varphi: T^{*} Y \rightarrow T^{*} \widetilde{Y}$ is a cotangent map to $\varphi$.

Example 2. An example of $\mathcal{F}^{2} \mathcal{M}_{m_{1}, m_{2}, n_{1}, n_{2}}$-natural operator

$$
D: Q_{\text {proj-proj }}^{\tau} \rightsquigarrow\left(T^{*}, \otimes^{3} T^{*}\right)
$$

is a family of operators $D^{1}=\left(D_{Y}^{1}\right)$,

$$
D_{Y}^{1}: Q_{\text {proj-proj }}^{\tau}(Y) \rightarrow C_{Y}^{\infty}\left(T^{*} Y, \otimes^{3} T^{*} Y\right),
$$

$D^{1}(\nabla): T^{*} Y \rightarrow \otimes^{3} T^{*} Y$ given by $D_{Y}^{1}(\nabla)(\omega)=\omega \otimes \omega \otimes \omega$, where $\omega \in T_{y}^{*} Y$, $y \in Y, Y \in \operatorname{Obj}\left(\mathcal{F}^{2} \mathcal{M}_{m_{1}, m_{2}, n_{1}, n_{2}}\right), \nabla \in Q_{\text {proj-proj }}^{\tau}(Y)$.

Another example of $\mathcal{F}^{2} \mathcal{M}_{m_{1}, m_{2}, n_{1}, n_{2}}$-natural operator

$$
D: Q_{\text {proj-proj }}^{\tau} \rightsquigarrow\left(T^{*}, \otimes^{3} T^{*}\right)
$$

is a family of operators $D^{2}=\left(D_{Y}^{2}\right)$,

$$
D_{Y}^{2}: Q_{\text {proj-proj }}^{\tau}(Y) \rightarrow C_{Y}^{\infty}\left(T^{*} Y, \otimes^{3} T^{*} Y\right),
$$

$D^{2}(\nabla): T^{*} Y \rightarrow \otimes^{3} T^{*} Y$ given by $D_{Y}^{2}(\nabla)(\omega)=\left\langle R_{y}(\nabla), \omega\right\rangle$, where $R(\nabla)$ is the curvature tensor of $\nabla, \omega \in T_{y}^{*} Y, y \in Y, Y \in \operatorname{Obj}\left(\mathcal{F}^{2} \mathcal{M}_{m_{1}, m_{2}, n_{1}, n_{2}}\right)$, $\nabla \in Q_{\text {proj-proj }}^{\tau}(Y)$.

Example 3. An example of $\mathcal{F}^{2} \mathcal{M}_{m_{1}, m_{2}, n_{1}, n_{2}}$-natural operator

$$
D: Q_{\text {proj-proj }}^{\tau} \rightsquigarrow\left(T^{*}, \otimes^{2} T^{*} \otimes T\right)
$$

is a family of operators $D^{3}=\left(D_{Y}^{3}\right)$,

$$
D_{Y}^{3}: Q_{\text {proj-proj }}^{\tau}(Y) \rightarrow C_{Y}^{\infty}\left(T^{*} Y, \otimes^{2} T^{*} Y \otimes T Y\right),
$$

$D^{3}(\nabla): T^{*} Y \rightarrow \otimes^{2} T^{*} Y \otimes T Y$ given by $\left\langle D_{Y}^{3}(\nabla)(\omega), v_{1} \otimes v_{2}\right\rangle=\left\langle\omega, v_{1}\right\rangle v_{2}$, where $\omega \in T_{y}^{*} Y, v_{1}, v_{2} \in T_{y} Y, y \in Y, Y \in \operatorname{Obj}\left(\mathcal{F}^{2} \mathcal{M}_{m_{1}, m_{2}, n_{1}, n_{2}}\right), \nabla \in$ $Q_{\text {proj-proj }}^{\tau}(Y)$. 
2. Some lemmas. The following lemma shows that the description of $\mathcal{F}^{2} \mathcal{M}_{m_{1}, m_{2}, n_{1}, n_{2}}$-natural operators $D: Q_{\text {proj-proj }}^{\tau} \rightsquigarrow Q T^{*}$ can be replaced by the description of $\mathcal{F}^{2} \mathcal{M}_{m_{1}, m_{2}, n_{1}, n_{2}}$-natural operators $D: Q_{\text {proj-proj }}^{\tau} \rightsquigarrow$ $\left(T^{*}, \otimes^{p} T^{*} \otimes \otimes^{q} T\right)$.

Lemma 1. There exists a bijection between the set of $\mathcal{F}^{2} \mathcal{M}_{m_{1}, m_{2}, n_{1}, n_{2}}$ natural operators $D: Q_{\text {proj-proj }}^{\tau} \rightsquigarrow Q T^{*}$ and the set of sequences $\left(D_{i}\right)_{i=1, \ldots, 8}$

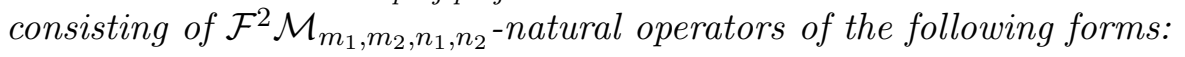

$$
\begin{aligned}
& D_{1}: Q_{\text {proj-proj }}^{\tau} \rightsquigarrow\left(T^{*}, T^{*} \otimes T^{*} \otimes T\right) \\
& D_{2}: Q_{\text {proj-proj }}^{\tau} \rightsquigarrow\left(T^{*}, T^{*} \otimes T^{*} \otimes T^{*}\right) \\
& D_{3}: Q_{\text {proj-proj }}^{\tau} \rightsquigarrow\left(T^{*}, T \otimes T^{*} \otimes T\right) \\
& D_{4}: Q_{\text {proj-proj }}^{\tau} \rightsquigarrow\left(T^{*}, T \otimes T^{*} \otimes T^{*}\right) \\
& D_{5}: Q_{\text {proj-proj }}^{\tau} \rightsquigarrow\left(T^{*}, T^{*} \otimes T \otimes T\right) \\
& D_{6}: Q_{\text {proj-proj }}^{\tau} \rightsquigarrow\left(T^{*}, T^{*} \otimes T \otimes T^{*}\right) \\
& D_{7}: Q_{\text {proj-proj }}^{\tau} \rightsquigarrow\left(T^{*}, T \otimes T \otimes T\right) \\
& D_{8}: Q_{\text {proj-proj }}^{\tau} \rightsquigarrow\left(T^{*}, T \otimes T \otimes T^{*}\right) .
\end{aligned}
$$

Proof. Let $\nabla \in Q_{\text {proj-proj }}^{\tau}(Y)$ be a projectable-projectable classical torsionfree linear connection on an $\left(m_{1}, m_{2}, n_{1}, n_{2}\right)$-dimensional fibred-fibred manifold $Y$. Let $v \in T_{y}^{*} Y, y \in Y$.

The connection $\nabla$ yields a decomposition of the tangent space $T_{v} T^{*} Y$ of $T^{*} Y$ at $v$ of the form

$$
T_{v} T^{*} Y=H_{v}^{\nabla} \oplus V_{v} T^{*} Y,
$$

where $H_{v}^{\nabla}$ is a $\nabla$-horizontal subspace and $V_{v} T^{*} Y$ is a vertical subspace.

We have an isomorphism $H_{v}^{\nabla} \cong T_{y} Y$ by the restriction of the differential $T_{v} \pi: T_{v} T^{*} Y \rightarrow T_{y} Y$ of the cotangent bundle projection $\pi: T^{*} Y \rightarrow Y$ to $H_{v}^{\nabla}$. Moreover, we have an isomorphism $V_{v} T^{*} Y \cong T_{y}^{*} Y$ by the standard isomorphism

$$
\left.T_{y}^{*} Y \ni \omega \rightarrow \frac{d}{d t}\right|_{0}(v+t \omega) \in T_{v} T^{*} Y=V_{v} T^{*} Y .
$$

Thus we have a decomposition

$$
T_{v} T^{*} Y \cong T_{y} Y \oplus T_{y}^{*} Y
$$

canonically depending on $\nabla$.

Consequently, we have a linear isomorphism

$T_{v}^{*} T^{*} Y \otimes T_{v}^{*} T^{*} Y \otimes T_{v} T^{*} Y \cong\left(T_{y} Y \oplus T_{y}^{*} Y\right)^{*} \otimes\left(T_{y} Y \oplus T_{y}^{*} Y\right)^{*} \otimes\left(T_{y} Y \oplus T_{y}^{*} Y\right)$ canonically depending on $\nabla$.

We have an isomorphism

$$
\left(T_{y} Y \oplus T_{y}^{*} Y\right)^{*} \cong T_{y}^{*} Y \oplus T_{y} Y
$$


by standard identifications

$$
(V \oplus W)^{*}=V^{*} \oplus W^{*} \quad \text { and } \quad V^{* *}=V,
$$

from linear algebra.

Thus we have the following linear isomorphism

$$
\begin{array}{r}
T_{v}^{*} T^{*} Y \otimes T_{v}^{*} T^{*} Y \otimes T_{v} T^{*} Y \cong\left(T_{y}^{*} Y \otimes T_{y}^{*} Y \otimes T_{y} Y\right) \oplus\left(T_{y}^{*} Y \otimes T_{y}^{*} Y \otimes T_{y}^{*} Y\right) \\
\oplus\left(T_{y} Y \otimes T_{y}^{*} Y \otimes T_{y} Y\right) \oplus\left(T_{y} Y \otimes T_{y}^{*} Y \otimes T_{y}^{*} Y\right) \oplus\left(T_{y}^{*} Y \otimes T_{y} Y \otimes T_{y} Y\right) \\
\oplus\left(T_{y}^{*} Y \otimes T_{y} Y \otimes T_{y}^{*} Y\right) \oplus\left(T_{y} Y \otimes T_{y} Y \otimes T_{y} Y\right) \oplus\left(T_{y} Y \otimes T_{y} Y \otimes T_{y}^{*} Y\right)
\end{array}
$$

canonically depending on $\nabla$.

Using the above isomorphism, for any $\mathcal{F}^{2} \mathcal{M}_{m_{1}, m_{2}, n_{1}, n_{2}}$-natural operator $D: Q_{\text {proj-proj }}^{\tau} \rightsquigarrow Q T^{*}$, we can define a sequence of eight $\mathcal{F}^{2} \mathcal{M}_{m_{1}, m_{2}, n_{1}, n_{2}}{ }^{-}$ natural operators $D_{1}, \ldots, D_{8}$ such as in Lemma 1, taking

$$
\left(D_{1}(\nabla)(v), \ldots, D_{8}(\nabla)(v)\right):=\left(D(\nabla)-\nabla^{T^{*}}\right)(v)
$$

for any $\nabla \in Q_{\text {proj-proj }}^{\tau}(Y), Y \in \operatorname{Obj}\left(\mathcal{F}^{2} \mathcal{M}_{m_{1}, m_{2}, n_{1}, n_{2}}\right), v \in T_{y}^{*} Y, y \in Y$, where $\nabla^{T^{*}}$ is the horizontal lift of $\nabla$ to $T^{*} Y$.

The difference $D(\nabla)-\nabla^{T^{*}}$ of linear connections $D(\nabla)$ and $\nabla^{T^{*}}$ means a tensor field of type $T^{*} \otimes T^{*} \otimes T$ on $T^{*} Y$.

Above relation (1) makes sense because it holds $\left(D(\nabla)-\nabla^{T^{*}}\right)(v) \in$ $T_{v}^{*} T^{*} Y \otimes T_{v}^{*} T^{*} Y \otimes T_{v} T^{*} Y$ and $\left(D_{1}(\nabla)(v), \ldots, D_{8}(\nabla)(v)\right) \in\left(\left(T_{y}^{*} Y \otimes T_{y}^{*} Y \otimes\right.\right.$ $\left.\left.T_{y} Y\right) \oplus \ldots \oplus\left(T_{y} Y \otimes T_{y} Y \otimes T_{y}^{*} Y\right)\right) \cong T_{v}^{*} T^{*} Y \otimes T_{v}^{*} T^{*} Y \otimes T_{v} T^{*} Y$, where $\cong$ is a linear isomorphism canonically depending on $\nabla$ describing above.

It is obvious that an assignment $D \mapsto\left(D_{i}\right)_{i=1, \ldots, 8}$ yields the bijection from Lemma 1.

Note that the description of natural operators $D_{1}, D_{4}$ and $D_{6}$ from Lemma 1 can be reduced to the description of operators of type $D_{1}$ since by obviously linear isomorphisms obtaining by permutations of factors

$$
T_{y}^{*} Y \otimes T_{y}^{*} Y \otimes T_{y} Y \cong T_{y} Y \otimes T_{y}^{*} Y \otimes T_{y}^{*} Y \cong T_{y}^{*} Y \otimes T_{y} Y \otimes T_{y}^{*} Y
$$

for any $Y \in \operatorname{Obj}\left(\mathcal{F}^{2} \mathcal{M}_{m_{1}, m_{2}, n_{1}, n_{2}}\right)$ and $y \in Y$ we have

Lemma 2. There exists the bijection between the set of $\mathcal{F}^{2} \mathcal{M}_{m_{1}, m_{2}, n_{1}, n_{2}}$ natural operators $D_{1}: Q_{\text {proj-proj }}^{\tau} \rightsquigarrow\left(T^{*}, T^{*} \otimes T^{*} \otimes T\right)$ and the set of $\mathcal{F}^{2} \mathcal{M}_{m_{1}, m_{2}, n_{1}, n_{2}}$ natural operators $D_{4}: Q_{\text {proj-proj }}^{\tau} \rightsquigarrow\left(T^{*}, T \otimes T^{*} \otimes T^{*}\right)$.

Similarly, there exists the bijection between the set of $\mathcal{F}^{2} \mathcal{M}_{m_{1}, m_{2}, n_{1}, n_{2}}$ natural operators $D_{1}: Q_{\text {proj-proj }}^{\tau} \rightsquigarrow\left(T^{*}, T^{*} \otimes T^{*} \otimes T\right)$ and the set of $\mathcal{F}^{2} \mathcal{M}_{m_{1}, m_{2}, n_{1}, n_{2}}$ natural operators $D_{6}: Q_{\text {proj-proj }}^{\tau} \rightsquigarrow\left(T^{*}, T^{*} \otimes T \otimes T^{*}\right)$.

Proof. The first bijection is of the form $D_{1} \mapsto D_{4}$, where $D_{4}(\nabla)(v):=$ $D_{1}(\nabla)(v), v \in T_{y}^{*} Y, y \in Y, \nabla \in Q_{\text {proj-proj }}^{\tau}(Y)$ modulo the identification $T_{y}^{*} Y \otimes T_{y}^{*} Y \otimes T_{y} Y \cong T_{y} Y \otimes T_{y}^{*} Y \otimes T_{y}^{*} Y$ of the form $\omega_{1} \otimes \omega_{2} \otimes \omega \mapsto \omega \otimes \omega_{1} \otimes \omega_{2}$.

The second bijection is analogous. 
Moreover, we show that $\mathcal{F}^{2} \mathcal{M}_{m_{1}, m_{2}, n_{1}, n_{2}}$-natural operators $D_{3}, D_{5}, D_{7}$ and $D_{8}$ from Lemma 1 are zero. It holds the following general fact.

Lemma 3. Let $p<q$. Then every $\mathcal{F}^{2} \mathcal{M}_{m_{1}, m_{2}, n_{1}, n_{2}}$-natural operator

$$
D: Q_{\text {proj-proj }}^{\tau} \rightsquigarrow\left(T^{*}, \otimes^{p} T^{*} \otimes \otimes^{q} T\right)
$$

is zero.

Proof. Let $\nabla \in Q_{\text {proj-proj }}^{\tau}(Y), Y \in \operatorname{Obj}\left(\mathcal{F}^{2} \mathcal{M}_{m_{1}, m_{2}, n_{1}, n_{2}}\right), v \in T_{y}^{*} Y, y \in$ $Y$. We have to show that $D(\nabla)(v)=0 \in \otimes^{p} T_{y}^{*} Y \otimes \otimes^{q} T_{y} Y$. By the $\mathcal{F}^{2} \mathcal{M}_{m_{1}, m_{2}, n_{1}, n_{2}}$-invariance of $D$ with respect to $\mathcal{F}^{2} \mathcal{M}_{m_{1}, m_{2}, n_{1}, n_{2}}$-charts we can assume $Y=\mathbb{R}^{m_{1}, m_{2}, n_{1}, n_{2}}, y=(0,0,0,0) \in \mathbb{R}^{m_{1}+m_{2}+n_{1}+n_{2}}$.

Then using the invariance of $D$ with respect to $\mathcal{F}^{2} \mathcal{M}_{m_{1}, m_{2}, n_{1}, n_{2}}$-maps (homotheties)

$$
\frac{1}{t} i d: \mathbb{R}^{m_{1}, m_{2}, n_{1}, n_{2}} \rightarrow \mathbb{R}^{m_{1}, m_{2}, n_{1}, n_{2}} \quad \text { for } t \neq 0,
$$

we get the condition

$$
D(\nabla)(v)=\left(\frac{1}{t}\right)^{q-p} D\left(\left(\frac{1}{t} i d\right)_{*} \nabla\right)(t v), \quad t \neq 0 .
$$

But the family $\left(\nabla_{t}\right)$ of projectable-projectable classical torsion-free linear connections given by

$$
\nabla_{t}:= \begin{cases}\left(\frac{1}{t} i d\right)_{*} \nabla, & t \neq 0 \\ \nabla_{0}, & t=0\end{cases}
$$

where $\nabla_{0}$ is the flat torsion-free linear connection (i.e. with zero Christoffel symbols), is smoothly parameterized because of the fact that $\nabla_{t}$ has

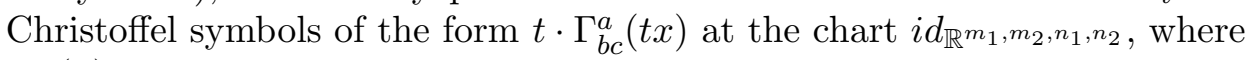
$\Gamma_{b c}^{a}(x)$ are the Christoffel symbols for $\nabla$.

Thus using the regularity of $D$ and taking $t \rightarrow \infty$, we get $D(\nabla)(v)=0$ since $\left(\frac{1}{t}\right)^{q-p}=t^{p-q} \rightarrow 0$ for $p<q$.

3. The main results. As the summary of Lemmas 1-3 we get the following main theorem.

Theorem 1. There exists the bijection between the set of $\mathcal{F}^{2} \mathcal{M}_{m_{1}, m_{2}, n_{1}, n_{2}}$ natural operators $D: Q_{\text {proj-proj }}^{\tau} \rightsquigarrow Q T^{*}$ and the set of sequences $\left(\widetilde{D}_{i}\right)_{i=1,2,3,4}$ consisting of $\mathcal{F}^{2} \mathcal{M}_{m_{1}, m_{2}, n_{1}, n_{2}}$-natural operators $\widetilde{D}_{1}, \widetilde{D}_{2}, \widetilde{D}_{3}: Q_{\text {proj-proj }}^{\tau} \rightsquigarrow$ $\left(T^{*}, T^{*} \otimes T^{*} \otimes T\right)$ and $\widetilde{D}_{4}: Q_{\text {proj-proj }}^{\tau} \rightsquigarrow\left(T^{*}, T^{*} \otimes T^{*} \otimes T^{*}\right)$.

More precisely, the system of operators $\left(\widetilde{D}_{i}\right)_{i=1,2,3,4}$ defines a new sequence of operators $\left(D_{i}\right)_{i=1, \ldots, 8}$ (of the type from Lemma 1) such as

$$
\begin{aligned}
& D_{1}:=\widetilde{D}_{1}, D_{4}:=\widetilde{D}_{2}, D_{6}:=\widetilde{D}_{3} \text { (modulo the bijection from Lemma 2) } \\
& D_{2}:=\widetilde{D}_{4}, D_{3}=0, D_{5}=0, D_{7}=0, D_{8}=0 .
\end{aligned}
$$


This system of operators $\left(D_{i}\right)_{i=1, \ldots, 8}$ defines the $\mathcal{F}^{2} \mathcal{M}_{m_{1}, m_{2}, n_{1}, n_{2}}$-natural operator D (by Lemma 1).

Lemma 3 shows that the above assignment $\left(\widetilde{D}_{i}\right)_{i=1,2,3,4} \mapsto D$ is a bijection.

Theorem 1 reduces the classification of $\mathcal{F}^{2} \mathcal{M}_{m_{1}, m_{2}, n_{1}, n_{2}}$-natural operators $D: Q_{\text {proj-proj }}^{\tau} \rightsquigarrow Q T^{*}$ to the classification of $\mathcal{F}^{2} \mathcal{M}_{m_{1}, m_{2}, n_{1}, n_{2}}$-natural operators $D: Q_{\text {proj-proj }}^{\tau} \rightsquigarrow\left(T^{*}, \otimes^{p} T^{*} \otimes \otimes^{q} T\right)$ for $p=2, q=1$ and $p=3$, $q=0$.

Definition 3. An $\mathcal{F}^{2} \mathcal{M}_{m_{1}, m_{2}, n_{1}, n_{2}}$-natural operator

$$
D: Q_{\text {proj-proj }}^{\tau} \rightsquigarrow \otimes^{p} T^{*} \otimes \otimes{ }^{q} T
$$

is an $\mathcal{F}^{2} \mathcal{M}_{m_{1}, m_{2}, n_{1}, n_{2}}$-invariant family of regular operators

$$
D=\left(D_{Y}\right): Q_{\text {proj-proj }}^{\tau}(Y) \rightarrow C_{Y}^{\infty}\left(\otimes^{p} T^{*} Y \otimes \otimes^{q} T Y\right)
$$

defined for every $Y \in \operatorname{Obj}\left(\mathcal{F}^{2} \mathcal{M}_{m_{1}, m_{2}, n_{1}, n_{2}}\right)$, where $Q_{\text {proj-proj }}^{\tau}(Y)$ is defined in Definition 1 and $C_{Y}^{\infty}\left(\otimes^{p} T^{*} Y \otimes \otimes^{q} T Y\right)$ means the set of smooth tensor fields on $Y$.

The $\mathcal{F}^{2} \mathcal{M}_{m_{1}, m_{2}, n_{1}, n_{2}}$-invariance of $D$ means almost the same as in Definition 1, i.e. $\varphi$-related connections are transformed into $\varphi$-related tensor fields.

Example 4. An example of an $\mathcal{F}^{2} \mathcal{M}_{m_{1}, m_{2}, n_{1}, n_{2}}$-natural operator

$$
D: Q_{\text {proj-proj }}^{\tau} \rightsquigarrow \otimes^{3} T^{*} \otimes T
$$

is a family $D=\left(R_{Y}\right)$ of operators

$$
R_{Y}: Q_{\text {proj-proj }}^{\tau}(Y) \rightarrow C_{Y}^{\infty}\left(\otimes^{3} T^{*} Y \otimes T Y\right)
$$

for any $Y \in \operatorname{Obj}\left(\mathcal{F}^{2} \mathcal{M}_{m_{1}, m_{2}, n_{1}, n_{2}}\right)$, where $R_{Y}(\nabla)$ is the curvature tensor of $\nabla$.

Theorem 2. Let $p \geq q, r:=p-q$. There exists the bijection between the set of all $\mathcal{F}^{2} \mathcal{M}_{m_{1}, m_{2}, n_{1}, n_{2}}$-natural operators $D: Q_{\text {proj-proj }}^{\tau} \rightsquigarrow\left(T^{*}, \otimes^{p} T^{*} \otimes\right.$ $\left.\otimes^{q} T\right)$ and the set of $(r+1)$-elements sequences $\left(D_{i}\right)_{i=0,1, \ldots, r}$ consisting of $\mathcal{F}^{2} \mathcal{M}_{m_{1}, m_{2}, n_{1}, n_{2}}$-natural operators $D_{i}: Q_{\text {proj-proj }}^{\tau} \rightsquigarrow \otimes^{p} T^{*} \otimes \otimes^{q} T \otimes S^{i} T$, i.e. $D_{i}: Q_{\text {proj-proj }}^{\tau} \rightsquigarrow \otimes^{p} T^{*} \otimes \otimes^{q+i} T$ and $D_{i}(\nabla)\left(w_{1}, \ldots, w_{p}, v_{1}, \ldots, v_{q+i}\right)$ is symmetric with respect to $v_{q+1}, \ldots, v_{q+i}$.

Schema of the proof. Consider any $\mathcal{F}^{2} \mathcal{M}_{m_{1}, m_{2}, n_{1}, n_{2}}$-natural operator

$$
D: Q_{\text {proj-proj }}^{\tau} \rightsquigarrow\left(T^{*}, \otimes^{p} T^{*} \otimes \otimes^{q} T\right) .
$$

Let $\nabla \in Q_{\text {proj-proj }}^{\tau}\left(\mathbb{R}^{m_{1}, m_{2}, n_{1}, n_{2}}\right)$ and $v \in T_{(0,0,0,0)}^{*} \mathbb{R}^{m_{1}, m_{2}, n_{1}, n_{2}}$. We are going to study $D(\nabla)(v)$.

By the non-linear Petree theorem (see [3]) we have

$$
D(\nabla)(v)=D(\widetilde{\nabla})(v),
$$


where $\widetilde{\nabla}$ is some projectable-projectable classical torsion-free linear connection on $\mathbb{R}^{m_{1}, m_{2}, n_{1}, n_{2}}$ with Christoffel symbols $\widetilde{\nabla}_{b c}^{a}$ being polynomials of degree $k$. Thus we have

$$
\widetilde{\nabla}_{b c}^{a}=\sum_{|\alpha| \leq k} \nabla_{b c ; \alpha}^{a} x^{\alpha}
$$

where $\nabla_{b c ; \alpha}^{a} \in \mathbb{R}$ and $x^{1}, \ldots, x^{m_{1}+m_{2}+n_{1}+n_{2}}$ is the usual fibred-fibred coordinate system on $\mathbb{R}^{m_{1}, m_{2}, n_{1}, n_{2}}$.

In short, we write $D(\nabla)(v)=D\left(\nabla_{b c ; \alpha}^{a}\right)(v)$.

Using the invariance of $D$ with respect to homotheties $\frac{1}{t} i d, t \neq 0$, we get the homogeneity condition

$$
t^{r} D\left(\nabla_{b c ; \alpha}^{a}\right)(v)=D\left(t^{|\alpha|+1} \nabla_{b c ; \alpha}^{a}\right)(t v) .
$$

By the homogeneous function theorem (see [3]) and by the invariance of $D$ with respect to $\mathcal{F}^{2} \mathcal{M}_{m_{1}, m_{2}, n_{1}, n_{2}}$-charts we get that $D(\nabla)(v)$ is a polynomial of degree not higher than $r:=p-q$ with respect to $v \in T_{y}^{*} Y, y \in Y$, for every $Y \in \operatorname{Obj}\left(\mathcal{F}^{2} \mathcal{M}_{m_{1}, m_{2}, n_{1}, n_{2}}\right)$ and $\nabla \in Q_{\text {proj-proj }}^{\tau}(Y)$.

Thus we have

$$
D(\nabla)(t v)=\sum_{i=0}^{r} D_{i}(\nabla)(v) t^{i}
$$

for some uniquely determined coefficients $D_{i}(\nabla)(v) \in \otimes^{p} T_{y}^{*} Y \otimes \otimes{ }^{q} T_{y} Y$.

For every $a \in \mathbb{R}$ we have

$$
D(\nabla)(t a v)=\sum_{i=0}^{r} D_{i}(\nabla)(a v) t^{i}
$$

and

$$
D(\nabla)(\operatorname{tav})=\sum_{i=0}^{r} D_{i}(\nabla)(v) a^{i} t^{i}
$$

hence we get

$$
D(\nabla)(a v)=a^{i} D_{i}(\nabla)(v)
$$

It means that $D_{i}(\nabla)(v)$ is a polynomial of degree $i$ with respect to $v$ and it can be identified with the corresponding element

$$
D_{i}(\nabla)(v) \in \otimes{ }^{p} T_{y}^{*} Y \otimes \otimes{ }^{q} T_{y} Y \otimes S^{i} T_{y} Y .
$$

Summarizing, for every $\mathcal{F}^{2} \mathcal{M}_{m_{1}, m_{2}, n_{1}, n_{2}}$-natural operator

$$
D: Q_{\text {proj-proj }}^{\tau} \rightsquigarrow\left(T^{*}, \otimes^{p} T^{*} \otimes \otimes^{q} T\right)
$$

we defined the sequence $\left(D_{i}\right)_{i=0,1, \ldots, r}$ consisting of $\mathcal{F}^{2} \mathcal{M}_{m_{1}, m_{2}, n_{1}, n_{2}}$-natural operators

$$
D_{i}: Q_{\text {proj-proj }}^{\tau} \rightsquigarrow \otimes^{p} T^{*} \otimes \otimes^{q} T \otimes S^{i} T .
$$


Conversely, analysing the above reasoning, one can see that every $\mathcal{F}^{2} \mathcal{M}_{m_{1}, m_{2}, n_{1}, n_{2}}$-natural operator $D: Q_{\text {proj-proj }}^{\tau} \rightsquigarrow\left(T^{*}, \otimes^{p} T^{*} \otimes \otimes^{q} T\right)$ can be reconstructed from the sequence $\left(D_{i}\right)_{i=0,1, \ldots, r}$ of operators

$$
D_{i}: Q_{\text {proj-proj }}^{\tau} \rightsquigarrow \otimes^{p} T^{*} \otimes \otimes^{q} T \otimes S^{i} T .
$$

\section{REFERENCES}

[1] Doupovec, M., Mikulski, W. M., On prolongation of higher order connections, Ann. Polon. Math. 102, no. 3 (2011), 279-292.

[2] Kolár, I., Connections on fibered squares, Ann. Univ. Mariae Curie-Skłodowska, Sect. A 59 (2005), 67-76.

[3] Kolář, I., Michor, P. W., Slovák, J., Natural Operations in Differential Geometry, Springer-Verlag, Berlin-Heidelberg, 1993.

[4] Kurek, J., Mikulski, W. M., On prolongations of projectable connections, Ann. Polon. Math. 101, no. 3 (2011), 237-250.

[5] Kurek, J., Mikulski, W. M., The natural liftings of connections to tensor powers of the cotangent bundle, AGMP-8 Proceedings (Brno 2012), Miskolc Mathematical Notes, to appear.

[6] Kuréš, M., Natural lifts of classical linear connections to the cotangent bundle, Suppl. Rend. Mat. Palermo II 43 (1996), 181-187.

[7] Mikulski, W. M., The jet prolongations of fibered-fibered manifolds and the flow operator, Publ. Math. Debrecen 59 (3-4) (2001), 441-458.

[8] Yano, K., Ishihara, S., Tangent and Cotangent Bundles, Marcel Dekker, Inc., New York, 1973.

Anna Bednarska

Institute of Mathematics

Maria Curie-Skłodowska University

pl. M. Curie-Skłodowskiej 1

20-031 Lublin

Poland

e-mail: bednarska@hektor.umcs.lublin.pl

Received June 6, 2012 